\title{
A novel Fingerprint Image Preprocessing Algorithm
}

\author{
Ming FU \\ Computer \& Communication \\ Engineering Institute, \\ Changsha University of \\ Science \& Technology, \\ Changsha 410114, China
}

\author{
Jun HUANG \\ Computer \& Communication \\ Engineering Institute, \\ Changsha University of \\ Science \& Technology, \\ Changsha 410114, China \\ Hajun219@163.com
}

\author{
Jun XU \\ College of Information \\ Science and Engineering, \\ Hunan University, \\ Changsha 410082, China \\ xujun5811487@163.com
}

\begin{abstract}
Based on analyzing and experimenting a large number of the existing fingerprint image preprocessing algorithm, this paper presents a novel high-quality fingerprint image preprocessing algorithm. Taking full account of the inherent characteristics of the fingerprint image, the algorithm arranges overall features and local detail. The experiments show that it is a simple, efficient algorithm which can improve the quality of the fingerprint image, and lay a good foundation for the subsequent processing.
\end{abstract}

Keywords-Fingerprint Recognition; preprocessing; thinning

\section{INTRODUCTION}

Nowadays, Automated Fingerprint Identification System (AFIS) is to achieve recognition by the characteristics of the fingerprint matching ratio certification. It mainly related to: fingerprint image acquisition, the fingerprint image preprocessing, fingerprint image feature extraction, saving and comparing $^{[1]}$. Due to various reasons, the collected fingerprint image will contain a lot of noise and the accuracy of the fingerprint image feature extraction is heavily dependent on the quality of the fingerprint image, therefore fingerprint image preprocessing must be carried out before the feature extraction. For an original noisy fingerprint image, the purpose of preprocessing is: removing noise in the original image and turning it into a clear binary point chart, then fingerprint feature is easy to be correctly extracted ${ }^{[2]}$. The purpose of preprocessing is taking advantage of a variety of image processing techniques to remove image noise and recovering fingerprint ridge texture characteristics of the fingerprint image, so the preprocessing is the foundation and key part, it directly affects the result of feature extraction and matching. In the preprocessing process, it is necessary to keep the true characteristics without loss and reduce the variety of redundant information. Based on analyzing and experimenting a large number of the existing fingerprint image preprocessing algorithm, this paper presents a novel high-quality fingerprint image preprocessing algorithm. This paper includes four mainly parts: background extraction, smoothing, binarization and thinning ${ }^{[3]}$.

\section{BACKGROUND EXTRACTION}

Generally speaking, the collected fingerprint image, always unavoidably includes background region. The aim of separation fingerprint image from the background is to determine the effective area. It can be pertinently processed, which saves the processing time and ensures the treatment effect. This paper uses a novel adaptive local threshold image separation method to solve this problem. Based on making full use of the thought that fingerprint ridge along and vertical flow of grey level variance size are obviously different, this method uses the variance calculation of typical block size across $1 \sim 2$ grain line distance. Considering in different contrast conditions, the calculated variance of gray value change is big, in order to enable the selection of threshold value to meet local variance change. It is necessary to select suitable gray variance threshold value for each small piece gray contrast and partition the collected whole picture of fingerprint image $\mathrm{e}^{[4]}$. For of $320 * 320$ pixels size, the image is divided into twenty $20 * 20$ block, each size for $16 * 16$ pixels.

Selecting an initial threshold $T_{d}$ and reasonable gray contrast size $C_{d}$, then calculating each fingerprint image gray variance $\operatorname{Var}(k, l)$ and gray contrast $C(k, l)$.

$$
\begin{aligned}
& \operatorname{Var}(k, l)=\frac{1}{w \times w} \\
& \sum_{i=k \times w}^{(k+1) \times w} \sum_{j=l \times w}^{(l+1) \times w}(G(i, j)-\operatorname{Aver}(k, l))^{2} \\
& C(k, l)=\operatorname{LMax}(k, l)-\operatorname{LMin}(k, l)
\end{aligned}
$$

Where $G(i, j)$ denotes gray value of $(i, j)$, $\operatorname{LMax}(k, l), \operatorname{LMin}(k, l)$ denote minimum and maximum gray value of the $(k, l)$ respectively. $\operatorname{Aver}(k, l)$ denotes gray mean of this piece.

$$
\operatorname{Aver}(k, l)=(\operatorname{LMax}(k, l)-\operatorname{LMin}(k, l)) / 2
$$

Then each sub-block of threshold value is:

$$
\begin{aligned}
& T_{d}(k, l)=T_{d}+\operatorname{sign}\left(C(k, l)-C_{d}\right) \\
& \frac{\left(C(k, l)-C_{d}\right)^{2}}{C_{d}{ }^{2}} X T_{d} / 2
\end{aligned}
$$


If $\operatorname{Var}(k, l)>T_{d}(k, l)$, the $(k, l)$ piece is the fingerprint image area, or for the background region

\section{SMOOTHING}

Front image background has been extracted, so only prospect area needs smooth processing. In many image smoothing technique, spatial smoothing has been developed very active. After decades of exploration, there are a variety of effective smoothing algorithms. But they all have a common shortage: when algorithm smoothes noise, it also blurs the image boundary and details. In view of the above problem, this article adopts the smoothing algorithm of the literature [5], the experiment proved that this algorithm using bar template and a square plate to process the above problems indeed gets good effect. As shown in Figure.3.

\section{BINARIZATION}

Fingerprint image binarization is the binary image using 0 and 1 , the pixel value zero devotes that the fingerprint image ridge line area, and pixel value 1 devotes valley line area. This method not only reserves the fingerprint ridge characteristics, but also removes a lot of adhesion, noise and eliminates false details information. The traditional fingerprint image binarization methods mainly includes: fixed threshold method, local adaptive method, iterative dynamic threshold method, etc. The basic idea is using the grey of images information to determine a threshold value. The image gray scale which is greater than the threshold value of the pixels is taken as 1 , and below the threshold value of the pixels is taken as zero. But in actual fingerprint identification system, the traditional binarization method has some problems: processing speed can't keep up with the actual requirements, or it does not apply to low contrast or fuzzy image area, the effect is often not good. In view of the above reasons, this paper adopts dynamic threshold and fill method to binarize.

The main idea of the algorithm is [6]: the fingerprint image is divided into mutually disjoint several small windows, calculating the average gray level of related pixel in each window. Then chosing $P \pm \partial$ devotes the threshold value $T$ of the window. $\partial$ is empirical value and $\partial \in[10,30]$.Taking the size constant of the window occupies very important position. After several tests, in this paper, $13 * 13$ of the windows has been adopted for processing the segmentation image. For each pixel being processed, the gradation is larger than the threshold value is taken to set its gray value is 1 , and is 0 otherwise. Against the phenomenon of "white hole" or "black hole" After processing the binary image may appear, in the paper, $3 * 3$ of the window are used to judgments and fill each pixel.

The algorithm is described as follows ${ }^{[6]}$ : a) the entire image is divided into $13 * 13$ disjoint, window size is $w^{*} w$, Where $w$ is taken 13 in this article.

b) The center pixel $A(6,6)$ in each of the small window is for the processing element. Calculation the sum of all pixel gray value, which around and belong to A, making $P=\operatorname{sum} / 169, T=P \pm \partial$.

c) Calculate the gray value of $a$, if the value is greater than $T$, It is given as 1 , is 0 otherwise.

d) When the cycle is complete, taken the window of $3 * 3$ as $B$ and the center point $(1,1)$ as the processing element $b$ If the $b(x-1, y-1)$ 、 $\begin{array}{lcc}b(x-1, y) 、 & b(x-1, y+1) 、 & b(x+1, y-1) \\ b(x+1, y) 、 & b(x+1, y+1) \text { Or } \quad b(x-1, y-1) \\ b(x, y-1) & \quad b(x+1, y-1) \\ b(x, y+1) & b(x+1, y+1) \text { all are black spots, }\end{array}$ $b(x, y+1)$
taking $b$ point for black spots. Conversely, if there are at least seven of the white points in the above-mentioned points, then $b$ is given as the white point.

Comparing Figure.4 and Figure.5, it can be seen the proposed algorithm is still doing very reasonable treatment in poor image contrast.

\section{THINNING}

Thinning is a core aspect of fingerprint recognition preprocessing algorithm. Currently, there are many algorithms for thinning, such as OPTA, one of typical thinning algorithms, it is able to achieve the basic skeleton processing, however, the ridge line. After thinning it is not smooth. There are so many burrs that Characteristic values cannot very well be extracted. In addition, some additional thinning algorithms are also exist, this paper will not enumerate. Taking into account the strengths and weaknesses of the existing algorithm, this paper proposed a thinning algorithm based on the look-up table of eightneighborhood. This algorithm inherited template such as the OPTA thinning algorithm mode. However, the refinement process and the handling have been improved.

The detailed description is as follows ${ }^{[7]}$ : According to the above criterion, prior to making a table, from 0 to 255 from the 256 elements, and each element is either 0 or 1 . (Black dots) to be processed according to a certain point eight points adjacent to calculate the index value of the look-up table, if the elements of the check obtained in the table is 1 , indicates that the point can be deleted, or to retain.

a) In order to calculate the index value for lookup as shown in Figure.1 of the 8-neighborhood values: 


$$
\begin{array}{|l|l|l|}
\hline \text { P1 } & \text { P2 } & \text { P3 } \\
\hline \text { P4 } & \text { P0 } & \text { P5 } \\
\hline \text { P6 } & \text { P7 } & \text { P8 } \\
\hline
\end{array}
$$

Figure 1. Template for 8-neighborhood

$$
\begin{aligned}
& S=P 1 \times 2^{0}+P 2 \times 2^{1}+P 3 \times 2^{2}+P 4 \times 2^{3}+ \\
& P 5 \times 2^{4}+P 6 \times 2^{5}+P 7 \times 2^{6}+P 8 \times 2^{7}
\end{aligned}
$$

(Respectively for the values of 0 or 1 , there are 256 kinds of indexes and numerical 0-255 correspondence)

b) According to the above criterion to produce a table for eliminating, in fact, it is a onedimensional array with a capacity for 256, its Subscripts and index of the pixel values $S$ correspondence. When the array element value is 0 , which means the center of the array should be retained, is 1 , it means that should be removed. The arrays are defined as follows: Interasetable[256] =

$\{0,0,1,1,0,0,1,1,1,1,0,1,1,1,0,1,1,1,0,0,1$,

$1,1,1,0,0,0,0,0,0,0,1,0,0,1,1,0,0,1,1,1,1$,

$0,1,1,1,0,1,1,0,0,1,1,1,1,0,0,0,0,0,0,0,1$,

$1,1,0,0,1,1,0,0,0,0,0,0,0,0,0,0,0,0,0,0$,

$0,0,0,0,0,0,0,0,0,0,0,0,1,1,0,0,1,1,0,0$,

$1,1,0,1,1,1,0,1,0,0,0,0,0,0,0,0,0,0,0,0$,

$0,0,0,0,0,0,1,1,0,0,1,1,1,1,0,1,1,1,0,1,1$,

$1,0,0,1,1,1,1,0,0,0,0,0,0,0,1,0,0,1,1,0,0$,

$1,1,1,1,0,1,1,1,0,1,1,1,0,0,1,1,1,1,0,0,0,0$,

$0,0,0,0,1,1,0,0,1,1,0,0,0,0,0,0,0,0,0,0$,

$1,1,0,0,1,1,1,1,0,0,0,0,0,0,0,0,1,1,0,0,1$,

$1,0,0,1,1,0,1,1,1,0,0,0,1,1,0,0,1,1,1,0,1$,

$1,0,0,1,0,0,0\}$

c) From top to bottom, left to right, the binary image scanning, of every black spots: first determine the top and bottom of the black spots neighbors are black point, the point is not to be processed .In addition, if a black point is deleted, then skip the next neighbor, the next point, otherwise coded according to the 8neighborhood calculate the index value, the index value is the subscript of the array elements, find the corresponding subscript corresponding the elements to see if delete: If the black spots are removed, then skip it right neighborhood to deal with the next point.

d) From left to right, top to bottom, the binary image of the second scan, every black spots similar: first determine the left and right of the black spots neighbor. ranking, if we are all black spots, the point without processing, In addition, if a black point is deleted, then skip it right neighbors to deal with the next point; otherwise coded index value calculation 8 neighborhood, check the to eliminate table to see whether to delete: If the black spots delete, then skip the next neighbor to deal with the next point.

e) In this cycle, if the black points are deleted, skip c) Otherwise, the loop ends, thinning end

Results are shown after thinning in Figure.6. As you can see, the image after thinning by this algorithm has few burrs, the algorithm is also very good to extract the fingerprint skeleton. At the same time, it is best prepared for the next step Fingerprint Matching based on Minutiae.

\section{EXPERIMENT AND CONCLUSION}

In order to validate the effectiveness of the preprocessing algorithm, the experiments of feature point extraction and feature matching algorithm based on the similar triangles have been done in this chapter ${ }^{[8]}$. Based on a lot of experiments, the matlab7.0 simulations show: the preprocessing algorithm adopted in this paper can improve the fingerprint image processing effect and the quality of the fingerprint image significantly. In the same feature extraction algorithm and matching conditions, this preprocessing algorithm has higher recognition rate and shorter preprocessing time.

\section{ACKNOWLEDGEMENT}

This work is supported by Provincial Natural Science Foundation of Hunan under No. 11JJ3069;

Science Foundation of Hunan Province under Grant No. 2011SK3079.

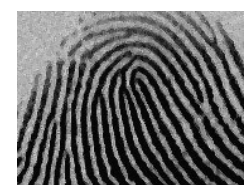

Figure 2. Original image
Figure 3. The image after processing

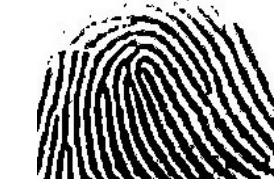

Figure 4. The image after processing by the conventional dynamic threshold method by the conventional dynamic threshold method
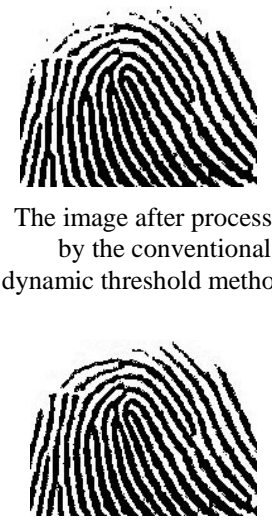

Figure 5. The image after processing by the dynamic threshold and filling method

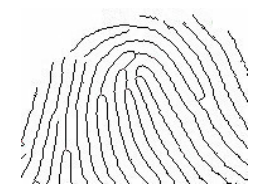

Figure 6. The image after thinning 


\section{REFERENCES}

[1] Lowe. D. G, "Distinctive image features from scale invariant key points," International Journal of Computer Vision, 60(2), 2004 , pp. 91-110.

[2] Xi ping. Luo, Jie. Tian, "Image Enhancement and Minutia Matching Algorithms in Automated Fingerprint Identification System," Journal of Software, 13(5), 2002,pp. 946-955.

[3] Yuan Yuan. Zhou, Cheng. Zhang, "Algorithms for fingerprint image preprocessing," Computer Applications, 24(9), Sept. 2004, pp.34-36

[4] Xiao Dong. Yang, Xin Bao. Ning, "Fingerprint Image Preprocessing Technique and Feature Extraction Algorithm," journal of nanjing university(natural science), 42(4), July .2006, pp.351-361.

[5] Xin e. You, "Fingerprint Information Extraction Research Based on the Orientation and Dynamic Threshold," Computer Simulation 29(8), Aug . 2012, pp.253-255.

[6] Chun Lei. Ji, Wei. Feng, "Dynamic Threshold with Hole Padding Algorithm for Fingerprint Image Binarization," Computer Simulation , 28(7), July. 2011, pp.258-261.

[7] Wei. Yang, Ke. Guo, "An Efficient Index Thinning Algorithm of Fingerprint Image Based on Eight Neighbourhood Points." Journal Of SiChuan University Of Science \& Engineering (Natural Science Edition), 21(2), Apr. 2008, pp.61-63.

[8] Ying. Chen, "Realization of automatic fingerprint identification system," Journal of zhejiang University of Science and Technology, 24(4), Aug. 2012, pp.303-309. 